\title{
LOG INTO ANDROID MOBILE TO FETCH THE DEVICE ORIENTED INFORMATION USING REMOTE ACCESS VIA A CLOUD
}

\author{
Vaishali Bhange $^{1}$, Nitin Singh Negi ${ }^{2}$, Dishant Mistry ${ }^{3}$ \\ ${ }^{1}$ Department of Computer Engineering, University of Pune, Maharashtra, INDIA \\ ${ }^{2}$ Department of Computer Engineering, University of Pune, Maharashtra, INDIA \\ ${ }^{3}$ Department of Computer Engineering, University of Pune, Maharashtra, INDIA
}

\begin{abstract}
This paper presents a platform for sharing device oriented mobile information via a local private cloud. The android application helps the user to fetch the device oriented information like missed call history, messages, battery status and GPS. Our system contains a website which helps the user to track all the required information through his personal account. Thus our system helps in making it easier to support new cloud services as output, and our android application helps in setting various triggers to push the device oriented information into the cloud.
\end{abstract}

Keywords: List Android, Device oriented information, Cloud.

\section{INTRODUCTION}

In today's era, Mobile devices are spreading at a remarkable rate. The usage of mobile devices is increasing rapidly. However nowadays not only mobile devices, but a user may also carry several devices simultaneously. Consider for instance a user may carry a mobile phone, an Android Tablet and a Laptop. In such cases information sharing between these devices becomes a prime goal. Also with the development of technologies, nowadays cloud computing has become the buzzword. The cloud provides easy and faster access to the data which makes it beneficial for the users. Additionally, users can access the required data and services anytime and anywhere. Thus with the development of cloud computing, the service providers no longer have to worry about the resources and resource management. Since it is managed by the cloud providers

The shared information is broadly categorized into two types, i.e the Device oriented information and the User oriented information. Sharing of information like mails, schedules is user based. But some information such as Missed call history, messages, sensor information is called as device oriented information. Here our system provides access to such device oriented information. In our project, our purpose is to create a platform that helps the user to share such device oriented information through a private cloud. The cloud is built using SaaS. The android application will push the data from the device to the cloud through a network connection. While the user can $\log$ into his account from the web browser to retrieve his desired data.
Our application provides the following functionalities:

1. Easy Access anytime and anywhere.

2. Security and Authorization

3. Device Independence

4. Remote Access

5. Easy Expansion

\section{RELATED WORKS}

There exists number of tools for pushing data into the cloud from smart phones. All these applications make use of internet. Some of them are as follows:

\subsection{Growl}

Growl is a local application that helps in sharing the information between the devices for which it first needs to be configured. When user receives some shared information on his device the growl's application which is activated in the device sends a notification to growl. This then shows a pop up to notify the user that the device received a shared information. Users can also set preferences so that the growl notifies accordingly. The application information is notified only to the devices inside the LAN.

\subsection{Context Watcher}

It is a context aware application which enables the users to remain updated with their information. This mobile application helps the end users to share their personal context data such as their speed, location, heartbeat with their mutual consent. For instance Family members, friends can stay updated about each other. 


\subsection{SECE}

It is a context aware platform. It helps in connecting different isolated services to be integrated so as to make it more powerful composite services. Thus it helps the users to create advanced services.

All the above applications are limited and restricted to share the information only on particular devices. However our goal is to create a platform such that this data is shared anytime and anywhere. Thus using the cloud services we can create a secured and effective environment for managing our data.

\section{ARCHITECTURE}

The prime focus of this system is to provide information sharing between devices. The user logs into the website through the web browser remotely to access the android phone via cloud. Thus the architecture is divided into three parts:

1] Website 2] Android application 3] Cloud Services

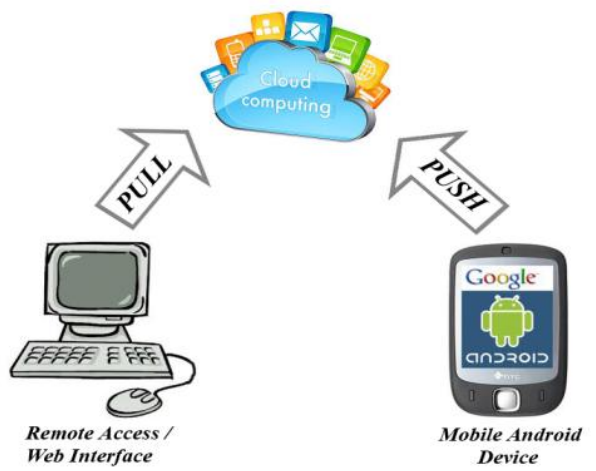

Fig 1: Architecture

\subsection{Website}

The website allows users to access their personal account. The user is assigned an ID and a password. With the help of this ID and password the user can log into the system anytime and from anywhere. The website provides an interface to the user to access the details. There will be certain options like check missed call history, messages, battery status, GPS etc. The user can click on the desired option and retrieve the required details.

\subsection{Android Application}

The android application helps in extracting all the device oriented information from the android phone. The android application updates the cloud via the network connection. The GPRS in android device should always remain switched ON so as to update the cloud and the web requests accordingly. The application updates the information on particular intervals on receiving requests from the cloud services.

\subsection{Cloud Services}

Cloud computing is used to describe a variety of different types of computing concepts. It involves connecting a large number of computers through networks such as internet.

It provides various services such as:

1] SaaS (Software as a service) which includes web browser, Mobile application, thin client, terminal emulator etc.

2] PaaS (Platform as a service) which includes execution runtime, database, web server, development tools etc.

3] IaaS (Infrastructure as a service) which includes virtual machines, servers, storage, load balancers, network etc.

In our project we make use of SaaS.

\section{OPERATIONS}

\subsection{Register}

Registration will help the user to create an account. With the help of which the user can access the necessary information like any other registration process The user will have to fill up the form which will provide him with an ID and password through which the user can access the phone's details.

\subsection{Login/logout}

When the user logs in the system it sets a trigger which will provide the user the information via a network connection. Once the data is retrieved user must log out for safety.

\subsection{Start Android Application}

The user with the application in the android phone must keep his application switched $\mathrm{ON}$ so that the data is periodically updated in the cloud via the network.

\subsection{Check}

This operation is performed after logging into the web interface. It enables the user to check the messages, missed call history, battery status, location of his phone.

\section{REQUIREMENTS}

\subsection{Technologies Used}

1. Android Smartphone with built in GPS and Android OS, version 2.1 and above.

2. Android Software Development Kit (SDK).

3. Eclipse using Android Development Toolkit (ADT).

4. Web services.

\subsection{Backend}

MySQL 


\subsection{Cloud}

Google App Engine Cloud Or any private cloud services.

\subsection{Operating System}

Windows XP or later versions

\section{CONCLUSIONS}

Thus our system provides a platform for sharing the device oriented information via a local private cloud. It enables the user to stay updated with his phone from a remote location. It provides great assistance in case of mobile theft. The user can track the location of his phone through a web browser with the help of cloud services. The user can access the required information anytime and from anywhere. Our system focuses on some important and fruitful technologies which may assist human race in easier interaction with the digital world.

\section{REFERENCES}

[1]. Platform for Pushing the Device-Oriented Information into a Cloud Kenji Morita, Department of Advanced Information Technology, Graduate School of Information Science and Electrical Engineering Kyushu University 744 Motooka Nishiku, Fukuoka 819-0395, Japan.

[2]. Michael Armbrust, Armando Fox, Rean Griffith, Anthony Joseph, Randy Katz, Andy Konwinski, Gunho Lee, David Patterson, Ariel Rabkin, Ion Stoica, and Matei Zaharia, Above the clouds: A Berkeley view of cloud computing, Technical Report UCB/EECS-2009-28, EECS Department, U.C. Berkeley,2009.

[3]. http://growl.info/

[4]. Johan Koolwaaij, Anthony Tarlano, Marko Luther, Petteri Nurmi, Bernd Mrohs, Agathe Battestini, Raju Vaidya, Context Watcher - Sharing Context Information in Everyday Life, IASTED International Conference on Web Technologies, Applications, and Services, Calgary, Canada, pp.12-21, 2006. 